\title{
AdipoRon, the first orally active adiponectin receptor activator, attenuates postischemic myocardial apoptosis through both AMPK-mediated and AMPK-independent signalings.
}

\author{
Yanqing Zhang \\ Shanxi Medical University \\ Jianli Zhao \\ Shanxi Medical University \\ Rui Li \\ Shanxi Medical University \\ WhymbetBieradd_additional works at: https://jdc.jefferson.edu/emfp \\ J'omas Jefferson University \\ Part of the Emergency Medicine Commons \\ kue:Xingshykanow how access to this document benefits you \\ Thomas Jefferson University
}

\section{Recommended Citation}

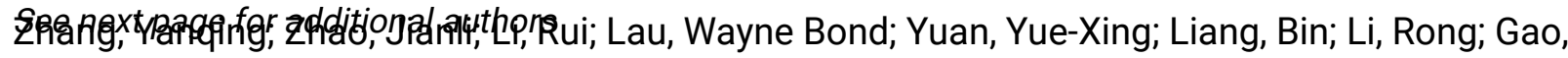
Er-He; Koch, Walter J.; Ma, Xin-Liang; and Wang, Ya-Jing, "AdipoRon, the first orally active adiponectin receptor activator, attenuates postischemic myocardial apoptosis through both AMPK-mediated and AMPK-independent signalings." (2015). Department of Emergency Medicine Faculty Papers. Paper 151.

https://jdc.jefferson.edu/emfp/151

This Article is brought to you for free and open access by the Jefferson Digital Commons. The Jefferson Digital Commons is a service of Thomas Jefferson University's Center for Teaching and Learning (CTL). The Commons is a showcase for Jefferson books and journals, peer-reviewed scholarly publications, unique historical collections from the University archives, and teaching tools. The Jefferson Digital Commons allows researchers and interested readers anywhere in the world to learn about and keep up to date with Jefferson scholarship. This article has been accepted for inclusion in Department of Emergency Medicine Faculty Papers by an authorized administrator of the Jefferson Digital Commons. For more information, please contact: JeffersonDigitalCommons@jefferson.edu. 


\section{Authors}

Yanqing Zhang, Jianli Zhao, Rui Li, Wayne Bond Lau, Yue-Xing Yuan, Bin Liang, Rong Li, Er-He Gao, Walter J. Koch, Xin-Liang Ma, and Ya-Jing Wang 
3

4

AdipoRon, the First Orally Active Adiponectin Receptor Activator, Attenuates Post-Ischemic Myocardial Apoptosis through both AMPK-Mediated and AMPK-Independent Signalings

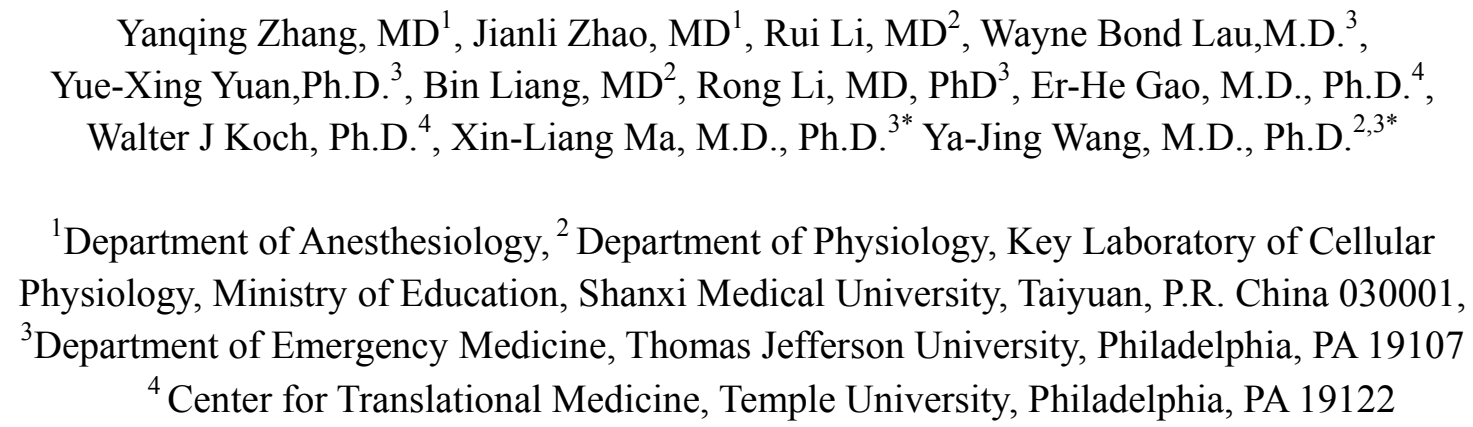


45 Adiponectin (APN) is a cardioprotective molecule. Its reduction in diabetes exacerbates 46 myocardial ischemia/reperfusion (MI/R) injury. Although APN administration in animals 47 attenuates $\mathrm{MI} / \mathrm{R}$ injury, multiple factors limit its clinical application. The current study 48 investigated whether AdipoRon, the first orally active molecule that binds APN receptors, may protect the heart against MI/R injury, and if so, to delineate the involved mechanisms. 50 Wild type (WT), APN knockout (APNKO), and cardiomyocyte specific-AMPK dominant negative (AMPK-DN) mice were treated with vehicle or AdipoRon $(50 \mathrm{mg} / \mathrm{kg}, 10$ minutes 52 prior to $\mathrm{MI}$ ) and subjected to $\mathrm{MI} / \mathrm{R}$ (30 minutes/3-24 hours). Compared to vehicle, oral administration of AdipoRon to WT mice significantly improved cardiac function, and 54 attenuated post-ischemic cardiomyocyte apoptosis determined by DNA ladder formation, TUNEL staining, and caspase-3 activation (all, $\mathrm{P}$ value $<0.01$ ). MI/R-induced apoptotic cell 56 death was significantly enhanced in mice deficient of either APN (APNKO) or AMPK (AMPK-DN). In APNKO mice, AdipoRon attenuated MI/R injury to the same degree observed in WT mice. In AMPK-DN mice, AdipoRon's anti-apoptotic action was partially inhibited, but not lost. Finally, AdipoRon significantly attenuated post-ischemic oxidative stress, as evidenced by reduced NADPH oxidase expression and superoxide production. Collectively, these results demonstrate for the first time that AdipoRon, an orally active APN receptor activator, effectively attenuated post-ischemic cardiac injury, supporting APN receptor agonists as a promising novel therapeutic approach treating cardiovascular complications caused by obesity-related disorders such as type 2 diabetes. 


\section{Abbreviations}

68 AAR area at risk

69 AdipoR1 Adiponectin Receptor-1

70 AdipoR2 Adiponectin receptor-2

71 AMPK 5'-adenosine monophosphate-activated protein kinase

72 APN Adiponectin

73 DHE dihydroethidium

74 GPCR G-protein coupled receptor

$75 \mathrm{MI} / \mathrm{R} \quad$ Myocardial ischemia/reperfusion

76 TUNEL terminal deoxynucleotidyl transferase-mediated dUTP nick-end labeling 
Ischemic heart disease is the single-most important cause of death in developed countries.

80 Accumulating evidence indicates that apoptosis, a gene-controlled programmed cell death

81 pathway, contributes significantly to post-ischemic cardiomyocyte death, suggesting

82 therapeutic interventions inhibiting apoptotic cell death may attenuate

83 ischemic/reperfusion-induced heart injury(10).

Adiponectin (APN) is an adipocyte-derived cytokine. Most adipokines (e.g., TNF $\alpha$ ) are

pro-inflammatory and significantly increased in diabetic patients. The majority of currently

published studies support APN as a potent cardiovascular protective molecule, and its levels

are markedly reduced in type 2 diabetic patients $(18 ; 26 ; 28)$. APN reduces oxidative/nitrative

stress, protects cells from apoptosis, inhibits leukocyte-endothelial interaction, and decreases

smooth muscle proliferation(9). Two APN receptors (AdipoR1 and AdipoR2) have been

cloned(37). Belonging to a new family of membrane receptors (i.e., the progestin and AdipoQ

receptor superfamily $)(7 ; 16 ; 29)$ predicted to contain seven transmembrane domains, the APN

receptors are topologically distinct from G-protein coupled receptors (GPCR). Although

numerous studies, done by others and us, demonstrate exogenous recombinant APN

supplementation significantly protects the heart from ischemia/reperfusion injury in

experimental animals, clinical APN application is limited due to multiple factors such as the

high cost of production.

al.(19) This synthetic small molecule is orally active, binds to and activates both AdipoR1 
molecule that effectively treats type 2 diabetes. However, whether AdipoRon may possess cardioprotective properties, attenuating post-ischemic cardiomyocyte death and improving cardiac function, have not been previously investigated.

Therefore, the aims of the present study were 1) to determine whether oral administration of AdipoRon may attenuate post-ischemic cardiomyocyte apoptosis and improve cardiac function recovery, and 2) if so, to investigate the underlying molecular mechanisms. 


\section{MATERIALS AND METHODS}

Adult male WT mice and APN knockout $\left(\mathrm{APN}^{-/}\right)$mice were purchased from the Jackson Laboratory (Bar Harbor, Maine). Cardiomyocyte-specific AMPK (5'-adenosine monophosphate-activated protein kinase)- $\alpha_{2}$ subunit mutant transgenic mice (AMPK-DN) were kindly provided by Dr. R Tina (University of Washington). Generation, breeding, phenotype characteristics, and genotyping of AMPK-DN mice $(>80 \%$ inhibition of cardiac AMPK activity) has previously been described in detail(36). The experiments were performed in adherence with the National Institutes of Health Guidelines on the Use of Laboratory Animals, and were approved by the Thomas Jefferson University Committee on Animal Care.

Myocardial ischemia/reperfusion: Mice were anesthetized with $2 \%$ isoflurane. MI/R was induced by temporarily exteriorizing the heart via a left thoracic incision, and placing a 6-0 silk suture slipknot around the left anterior descending coronary artery. Ten minutes before coronary occlusion, animals were randomized to receive either vehicle or AdipoRon (50 $\mathrm{mg} / \mathrm{kg}$, Calbiochem, Cat \# 509104) via a gavage tube. This dose was selected from previously published results demonstrating maximal blood concentration was achieved 30 minutes after a single oral dose of AdipoRon(19). After 30 minutes of MI, the slipknot was released. The myocardium was reperfused for either 3 hours (for all assays excluding cardiac functional measurement) or 24 hours (for cardiac functional assay). All assays were performed utilizing tissue from the ischemic/reperfused area, i.e. the area at risk (AAR) identified by Evans blue negative staining. Sham-operated control mice (Sham MI/R) underwent the same surgical procedure, except the suture placed under the left coronary artery was not tied. Cardiac function was determined by echocardiography and left ventricular catheterization methods 24 hours after reperfusion before thoracotomy, as described in our previous study(34). 
Assessment of cardiomyocyte apoptosis: Cardiomyocyte apoptosis was determined by DNA ladder formation, terminal deoxynucleotidyl transferase-mediated dUTP nick-end labeling

136 (TUNEL) staining, and caspase-3 activity as reported in our previous study(31). For the DNA fragmentation assay, total DNA was isolated with the Gentra Puregene Tissue DNA Isolation 138 Kit (QIAgen, Valencia, CA) per manufacturer's instructions. $10 \mu \mathrm{g}$ of DNA was loaded into $1391.8 \%$ agarose gel containing $0.5 \mu \mathrm{g} \mathrm{ml}^{-1}$ ethidium bromide. DNA electrophoresis commenced 140 at $60 \mathrm{~V}$ for 1-2 hours. DNA ladder formation, a "hallmark" of tissue apoptosis, was visualized under ultraviolet light, and photographed for permanent documentation.

142 TUNEL staining was performed via In Situ Cell Death Detection Kit (Roche Diagnostics $143 \mathrm{GmbH}$, Manheim, Germany) per manufacturer's protocol. In brief, cardiomyocytes from at least four random slides per block were evaluated immunohistochemically to determine the number and percentage of cells exhibiting apoptotic-positive staining. The slides were covered with the mounting medium containing DAPI for total nuclei detection. By $20 \mathrm{X}$ objective, the entire ischemic/reperfused area was digitally photographed with a QICAM-Fast Digital Camera mounted atop an Olympus BX51 Fluorescence Microscope. Total nuclei (blue) and the TUNEL positive nuclei (green) were counted by IP Lab Imaging Analysis Software (Version 3.5, Scanalytics, Fairfax, VA) with a custom-made script (by Mr. Ken Anderson, Bio Vision Technologies, North Exton, PA). The index of apoptosis (number of

152 TUNEL positive nuclei/total number of nuclei $\mathrm{x} 100$ ) was automatically calculated and exported to Microsoft Excel for further analysis. Results from different fields taken from the same animal were averaged and counted as 1 sample. The caspase- 3 activity was determined 155 by utilizing the fluorogenic substrate DEVD-7-amino-4-trifluoromethyl-coumarin (AFC). 
156 Briefly, cells or mouse heart tissue were lysed by $1 \mathrm{X}$ caspase-3 lysis buffer (50 mM HEPES, $157 \mathrm{pH} 7.4,0.1 \%$ CHAPS 3-[(3-cholamidopropyl)dimethylammonio]-1-propanesulfonate, $5 \mathrm{mM}$

158 DTT, $0.1 \mathrm{mM}$ EDTA, 0.1\% Triton X-100), and total protein concentration was determined by 159 the Bradford method (Bio-Rad). To each well of a 96-well plate, supernatant containing 50 $160 \mu \mathrm{g} / 50 \mu \mathrm{l}$ of protein was loaded and incubated with $3.645 \mu \mathrm{g}$ of Ac-DEVD-AFC (Biomol, 161 P-409) in 50 $\mu \mathrm{l} 2 \mathrm{X}$ Assay buffer (100 mM HEPES, 200mM NaCl, 0.2\% CHAPS, 2mM 162 EDTA, $10 \%$ glycerol, $10 \mathrm{mM}$ DTT, $\mathrm{pH} 7.4$ ) at $37^{\circ} \mathrm{C}$ for 1.5 hours. AFC was cleaved from 163 Ac-DEVD-AFC by activated caspase-3, and the free AFC was quantified with Spectra Max 164 M5 fluorescence microplate reader (excitation wavelength, $400 \mathrm{~nm}$; emission wavelength, $165508 \mathrm{~nm}$, Molecular Devices, Sunnyvale, CA) by AFC standard curve (Biomol, KI-108). 166 Caspase-3 activity was expressed as nanomoles of AFC formation per hour per milligram of 167 protein.

168 Quantification of superoxide production: Myocardial superoxide content (in the area-at-risk) 169 was determined by lucigenin-enhanced luminescence as previously described(34). 170 Approximately $30 \mathrm{mg}$ protein of ischemic left ventricular region was separated, immediately 171 minced, and incubated in $5 \mathrm{ml}$ of oxygen-equilibrated Krebs-Henseleit solution containing 10 $172 \mathrm{mM}$ HEPES-NaOH ( $\mathrm{pH}$ 7.4) for 20 minutes at room temperature. The samples were placed 173 into glass tubes containing $10 \mu \mathrm{M}$ lucigenin in a final volume of $1 \mathrm{ml} \mathrm{Krebs-Henseleit}$ 174 solution. Superoxide production was expressed as relative light units (RLU) per second per $175 \mathrm{mg}$ heart weight (RLU/s/mg wet tissue). The cellular origin of reactive oxygen species was 176 determined by dihydroethidium (DHE) staining per manufacturer's protocol (Molecular 177 Probes, Carlsbad, CA).

178 Western blot analysis: Proteins were separated on SDS-PAGE gels, transferred to 
nitrocellulose membranes, and incubated with primary antibodies against Acetyl-CoA carboxylase (ACC) phosphorylated ACC (pACC), gp91 ${ }^{\text {phox }}$ or GAPDH (Cell Signaling Technology, Danvers, MA) followed by HRP-conjugated secondary antibody. The blot was developed with a Supersignal Chemiluminescence detection kit (Pierce, Rockford, IL). The band was visualized by a Kodak Image Station 4000R Pro (Rochester, NY).

Determination of cardiac function: Cardiac function was determined by echocardiography and left ventricular (LV) catheterization methods 24 hours after reperfusion, prior to chest reopening, as described previously $(31 ; 32)$.

Statistical Analysis: All data in the text and figures are presented as means \pm S.E. of $n$ independent experiments. Hemodynamic data were analyzed by two-way ANOVA. All other data were analyzed by one-way ANOVA followed by the Bonferroni correction for post hoc $t$ tests (GraphPad Prism, San Diego, CA). Probabilities of 0.05 or less with Bonferroni correction were considered statistically significant. 


\section{RESULTS}

\section{AdipoRon treatment significantly improved cardiac functional recovery after reperfusion:}

Myocardial ischemia/reperfusion causes severe cardiac functional impairment 24 hours after reperfusion (Figure 1 and Figure 2, WT). Treatment with AdipoRon significantly improved cardiac functional recovery as evidenced by improved maximal positive and negative $\mathrm{dP} / \mathrm{dt}$ (Figure 1) and increased left ventricular ejection fraction (EF\%, Figure $2 \mathrm{WT})$. MABP was slightly decreased in the MI/R group 24 hours after reperfusion in comparison to the sham MI group. However, the difference was not statistically different. There was no difference in heart rate among the three groups studied (data not shown).

AdipoRon significantly inhibited post-MI apoptosis: To determine the cellular mechanism responsible for cardioprotective effect of AdipoRon, we first determined the effect of AdipoRon treatment on cardiomyocyte apoptotic death by DNA ladder formation, a hall marker of apoptotic cell death. In myocardial tissue from sham MI hearts, no DNA ladder was detected (Figure 3A WT, lanes 1). In contrast, the formation of DNA nucleosome ladders was clearly detected in myocardial tissues obtained from MI/R hearts receiving only vehicle (Figure 3A WT, lanes 2). Most importantly, hearts treated with AdipoRon exhibited markedly decreased DNA fragmentation (Figure 3A WT, lane 3).

To determine the effect of AdipoRon on apoptosis in a quantitative manner, caspase-3 activation and TUNEL staining were performed. AdipoRon treatment markedly reduced ischemia/reperfusion-induced caspase-3 activation (Figure 3B WT). In sham MI hearts, an extremely low level of TUNEL positive cells (Figure 4 WT) was observed. In contrast, tissues from ischemic-reperfused hearts receiving only vehicle manifested prevalent 
217 TUNEL-positive nuclei (Figure $4 \mathrm{WT}$ ). AdipoRon treatment reduced the number of

218 TUNEL-positive cells (Figure 4 WT).

219 Enhanced cardiomyocyte apoptosis in APN deficient mice is rescued by AdipoRon

220 administration: To determine whether AdipoRon is effective in rescuing the heart from 221 enhanced MI/R injury in APN deficient animals, the effect of AdipoRon on cardiac 222 dysfunction and cardiomyocyte apoptosis was determined in $\mathrm{APN}^{-/}$mice. AdipoRon 223 significantly improved cardiac function (Figure 1, Figure 2, $\mathrm{APN}^{-/}$) and reduced post-MI 224 cardiomyocyte apoptosis, as evidenced by attenuated ladder formation (Figure $3 \mathrm{~A}, \mathrm{APN}^{-/}$), 225 reduced caspase-3 activity (Figure $3 \mathrm{~B}, \mathrm{APN}^{-/}$), and decreased TUNEL positive cells (Figure $\left.2264, \mathrm{APN}^{-/}\right)$.

227 Anti-apoptotic effect of AdipoRon is attenuated but not lost in AMPK-DN mice: Compared 228 to WT, cardiac dysfunction and apoptotic cell death caused by MI/R was increased in the 229 AMPK-DN heart. We have reported previously the cardioprotective effects of APN are only 230 partially mediated by AMPK activation(34). In similar fashion, the beneficial effects of 231 AdipoRon upon cardiac dysfunction are blunted (Figure 1 and Figure 2, AMPK-DN vs. WT) 232 but not lost in AMPK-DN mice. Similarly, the anti-apoptotic effect of AdipoRon is partially 233 blocked but not completely lost in AMPK-DN mice. Specifically, administration of 234 AdipoRon reduced caspase- 3 activation by $39 \%$ (compared to $73 \%$ reduction in WT mice, 235 Figure 3 ) and TUNEL staining by $33 \%$ (comparing to $50 \%$ reduction in WT mice, Figure 4 ) 236 in AMPK-DN mice. In the AMPK-DN heart, there was complete blockade of 237 AdipoRon-induced phosphorylation of ACC (pACC, Figure 5A). Therefore, the remaining 238 portion of anti-apoptotic effect of AdipoRon in AMPK-DN mice can be attributed to AMPK 
independent signaling mechanisms.

\section{AdipoRon significantly reduced NADPH oxidase expression and inhibited superoxide}

241 production in ischemic/reperfused heart: Our previous study demonstrated the anti-oxidant

242 effect of APN is not mediated by AMPK(34). To determine whether AdipoRon may have any 243 anti-oxidant effect (potentially contributive to the remaining anti-apoptotic effect of 244 AdipoRon observed in AMPK-DN mice), the effect of AdipoRon upon post-ischemic 245 superoxide production and gp91 ${ }^{\text {phox }}$ (the primary sub-form of NADPH oxidase expressed in 246 adult cardiomyocytes) expression were determined in AMPK-DN mice. As summarized in 247 Figure 5, AdipoRon administration significantly reduced superoxide production assessed by 248 DHE staining (B) and lucigenin-enhanced luminescence assay (C). Moreover, AdipoRon 249 treatment significantly attenuated ischemia/reperfusion induced gp9 ${ }^{\text {phox }}$ overexpression (D). 250 


\section{Discussion}

Early reperfusion after coronary occlusion remains the most effective means of limiting ischemic myocardial injury. However, evidence from animal studies, as well as clinical observations, demonstrates reperfusion itself may cause additional cell death, defined as “reperfusion injury"(6). Strong epidemiological evidence suggests type-2 diabetes not only causes coronary vascular injury thereby increasing ischemic heart disease prevalence, but also exacerbates cardiac injury after ischemia/reperfusion insult in these patients $(4 ; 13 ; 17)$. Adiponectin (APN) is a protein hormone primarily produced by adipocytes (20). In contrast to the majority of adipokines (such as TNF $\alpha$ ), which are pro-inflammatory, and significantly increased in diabetic patients, APN is markedly reduced in diabetic patients, and is potently protective of the vasculature $(18 ; 26 ; 28)$. Plasma APN levels significantly decrease after tissue injury, such as acute lung injury caused by ovalbumin challenge(27). Numerous epidemiological studies reveal the association between hypoadiponectinemia and increased cardiovascular disease risk in obesity and diabetes $(8 ; 12 ; 15 ; 38)$. Additionally, recent clinical observations demonstrate post-MI plasma APN levels correlate positively with myocardial salvage index and ejection fraction recovery(23). Persistent plasma hypoadiponectinemia post myocardial infarction is predictive of future adverse cardiac events(2). Moreover, recent experimental studies demonstrate that myocardial reperfusion injury is significantly enhanced in APNKO mice. Replenishment of recombinant APN in APNKO mice was cardioprotective and fully rescued phenotypic alteration(20-22; 24). Importantly, multiple investigations [including the seminal study by Walsh and colleagues(25), a large animal model study by Kondo and colleagues (14), and our recent study (31)] have documented APN administration 
in WT mice and pigs significantly reduces infarct size and improves cardiac function. Despite clear experimental evidence that supplementation of recombinant human APN exerts significant anti-diabetic and cardioprotective actions, APN's complex quaternary structure and rapid turnover are major disadvantages to producing and administering APN in the requisite amounts for appropriate clinical care. Thus, the field has been awaiting the advent of low molecular weight APN receptor agonists capable of overcoming such hindrances.

In an effort to identify small synthetic molecules capable of activating the APN receptors (AdipoR1 and AdipoR2), Kadowaki and colleagues recently screened a compound library, and identified several molecules activating APN receptors, but focused their in-depth analysis upon one, “AdipoRon.”(19) AdipoRon binds, at a low micromolar concentration, to both AdipoR1 and AdipoR2. Like APN, it activates AMPK in cultured mammalian cells, an enzyme involved in many metabolic processes including insulin release, lipid synthesis inhibition, and glucose uptake stimulation. It also activates the transcriptional coactivator peroxisome proliferator-activated receptor gamma coactivator 1-alpha (PGC1 $\alpha$ ), which boosts mitochondrial proliferation and energy metabolism. Like APN, AdipoRon improved glucose metabolism, lipid metabolism, and insulin sensitivity in both cultured cells and mice, via APN receptor-dependent mechanisms. When $d b / d b$ mice fed a high-fat diet were treated with AdipoRon, their metabolic improvements extended their life span. Furthermore, AdipoRon administration to chow-fed wild-type mice augmented exercise endurance capacity. Their study convincingly demonstrated the viable strategy of targeting adiponectin receptors with low molecular weight agonists. 
hypoadiponectinemia to enhanced cardiovascular injury in the diabetic population. We and others have previously demonstrated $\mathrm{MI} / \mathrm{R}$ injury is significantly exacerbated in APN deficient mice, a phenotype fully rescued by recombinant APN replenishment. In the current study, AdipoRon administration rescued the pathological cardiac phenotype in APN deficient mice, similar to our previous report concerning APN administration(31). Moreover, our current study provides direct evidence that oral AdipoRon administration in WT mice also significantly improved post-ischemic cardiac function, as evidenced by increased $\pm \mathrm{dP} / \mathrm{dt}_{\max }$ recombinant APN, is biologically active in protecting heart from ischemia/reperfusion injury. ischemic/reperfused cardiomyocytes. contributes to post-ischemic cardiac dysfunction(3; 5). DNA ladder formation is highly specific for apoptotic cell death, but lacks sensitivity, and is difficult to quantify. In contrast, TUNEL staining of nuclei is extremely sensitive, but is less specific for apoptosis as some necrotic cells may stain TUNEL positive. Caspase- 3 activation is the final common pathway leading to caspase- 8 and caspase-9 induced apoptotic cell death. These three methods were used in combination to improve the accuracy and reliability of our results. AdipoRon reduced DNA ladder formation (Figure 3A), inhibited caspase-3 activation (Figure 3B), and decreased TUNEL staining (Figure 4), indicating AdipoRon possesses clear anti-apoptotic property in

AMPK was once considered the most important downstream molecule mediating APN 
biological function. The effect of cardiac-specific AMPK inhibition upon the anti-apoptotic effects of AdipoRon was determined. The beneficial effects of AdipoRon upon cardiac dysfunction (Figures 1,2) and apoptotis (Figures 3,4) after MI/R were clearly blunted in AMPK-DN mice. These results indicate AMPK activation contributes to the cardioprotective effect of AdipoRon. However, our results also clearly demonstrate the cardioprotective effect AdipoRon in AMPK-DN increased $\pm \mathrm{dP} / \mathrm{dt}_{\max }$ (1.35- and 1.34-fold), enhanced left ventricular ejection fraction (1.27-fold), reduced caspase-3 activation (39\%), and decreased TUNEL staining (33\%). That AdipoRon retained a significant portion of anti-apoptotic effect in AMPK-DN mice suggests the existence and contribution of mechanisms independent of AMPK signaling to AdipoRon-mediated anti-apoptotic function in the ischemic/reperfused heart. This result is consistent with our previous study showing that the cardioprotective effect of adiponectin is partially mediated by its AMPK-independent anti-nitrative action(35). Our current study provides supporting evidence the remaining anti-apoptotic action of by following three lines of evidence. First, oxidative stress plays critical causative roles in post-ischemic myocardial apoptosis and cardiac dysfunction(1; 30; 33); Second, the anti-oxidative effect of APN is AMPK-independent; and third, AdipoRon inhibits NADPH oxidase overexpression and superoxide overproduction in the ischemic/reperfused heart. In summary, our study demonstrated that the oral APN receptor agonist AdipoRon is effective in attenuating post-ischemic cardiac injury, indicating APN receptor agonists are a 
337 promising novel therapeutic approach for treating cardiovascular complications caused by 338 obesity-related disorders such as type 2 diabetes(11). 
Disclosures

341

None

342 
343

344

345

346

347

348

349

350

351

352

353

\section{Acknowledgement}

We are greatly appreciative of Mr. Nadan Wang in the Center for Translational Medicine, Thomas Jefferson University, for his expertise in evaluation of cardiac function by echocardiography. This research was supported by grants NIH HL-096686, HL-123404, American Diabetes Association 7-11-BS-93 (XLM), American Diabetes Association 1-14-BS-228, National Science Foundation of China 31322026 and 81170199 (YJW) and National Science Foundation of China 81270185 and 81470020 (JLZ). 
1. Ambrosio G, Zweier JL and Becker LC. Apoptosis is prevented by administration of superoxide dismutase in dogs with reperfused myocardial infarction. Basic Res Cardiol 93: 94-96, 1998. relation of MAPK activation to infarct size reduction by ischemic preconditioning in pigs. Am J Physiol 279: 1111-1119, 2000.

PA. Role of apoptosis in reperfusion injury. Cardiovasc Res 61: 414-426, 2004. infarction in dogs with experimental diabetes. Cardiovasc Res 27: 1908-1912, 1993.

5. Gao F, Tao L, Yan W, Gao E, Liu HR, Lopez BL, Christopher TA and Ma XL. Early anti-apoptosis treatment reduces myocardial infarct size after a prolonged reperfusion. Apoptosis 9: 553-559, 2004.

6. Garcia-Dorado D. Myocardial reperfusion injury: a new view. Cardiovasc Res 61: 363-364, 2004.

7. Garitaonandia I, Smith JL, Kupchak BR and Lyons TJ. Adiponectin identified as an agonist for PAQR3/RKTG using a yeast-based assay system. J Recept Signal Transduct Res 29: 67-73, 2009. 
9. Goldstein BJ, Scalia RG and Ma XL. Protective vascular and myocardial effects of adiponectin. Nat Clin Pract Cardiovasc Med 6: 27-35, 2009.

10. Gottlieb RA and Engler RL. Apoptosis in myocardial ischemia-reperfusion. Ann N Y Acad Sci 874: 412-426, 1999.

11. Holland WL and Scherer PE. Cell Biology. Ronning after the adiponectin receptors. Science 342: 1460-1461, 2013.

12. Hotta K, Funahashi T, Arita Y, Takahashi M, Matsuda M, Okamoto Y, Iwahashi H, Kuriyama H, Ouchi N, Maeda K, Nishida M, Kihara S, Sakai N, Nakajima T, Hasegawa K, Muraguchi M, Ohmoto Y, Nakamura T, Yamashita S, Hanafusa T and Matsuzawa Y. Plasma concentrations of a novel, adipose-specific protein, adiponectin, in type 2 diabetic patients. Arterioscler Thromb Vasc Biol 20: 1595-1599, 2000.

13. Jagasia D and McNulty PH. Diabetes mellitus and heart failure. Congest Heart Fail 9: 133-139, 2003.

\section{Kondo K, Shibata R, Unno K, Shimano M, Ishii M, Kito T, Shintani S, Walsh K, Ouchi N and} Murohara T. Impact of a single intracoronary administration of adiponectin on myocardial ischemia/reperfusion injury in a pig model. Circ Cardiovasc Interv 3: 166-173, 2010.

\section{Kumada M, Kihara S, Sumitsuji S, Kawamoto T, Matsumoto S, Ouchi N, Arita Y, Okamoto Y,} Shimomura I, Hiraoka H, Nakamura T, Funahashi T and Matsuzawa Y. Association of hypoadiponectinemia with coronary artery disease in men. Arterioscler Thromb Vasc Biol 23: 85-89, 
2003.

16. Kupchak BR, Garitaonandia I, Villa NY, Smith JL and Lyons TJ. Antagonism of human adiponectin receptors and their membrane progesterone receptor paralogs by TNFalpha and a ceramidase inhibitor. Biochemistry 48: 5504-5506, 2009.

17. Marfella R, D'Amico M, Di Filippo C, Piegari E, Nappo F, Esposito K, Berrino L, Rossi F and Giugliano D. Myocardial infarction in diabetic rats: role of hyperglycaemia on infarct size and early expression of hypoxia-inducible factor 1. Diabetologia 45: 1172-1181, 2002.

20. Ouchi N, Shibata R and Walsh K. Cardioprotection by adiponectin. Trends Cardiovasc Med 16: 141-146, 2006.

19. Okada-Iwabu M, Yamauchi T, Iwabu M, Honma T, Hamagami KI, Matsuda K, Yamaguchi M, Tanabe H, Kimura-Someya T, Shirouzu M, Ogata H, Tokuyama K, Ueki K, Nagano T, Tanaka A, Yokoyama S and Kadowaki T. A small-molecule AdipoR agonist for type 2 diabetes and short life in obesity. Nature 503: 493-499, 2013.

\section{Sam F, Duhaney TA, Sato K, Wilson RM, Ohashi K, Sono-Romanelli S, Higuchi A, De Silva DS,} Qin F, Walsh K and Ouchi N. Adiponectin deficiency, diastolic dysfunction, and diastolic heart failure. 


\section{Shibata R, Izumiya Y, Sato K, Papanicolaou K, Kihara S, Colucci WS, Sam F, Ouchi N and Walsh} K. Adiponectin protects against the development of systolic dysfunction following myocardial infarction. J Mol Cell Cardiol 42: 1065-1074, 2007.

23. Shibata R, Numaguchi Y, Matsushita K, Sone T, Kubota R, Ohashi T, Ishii M, Kihara S, Walsh K, Ouchi N and Murohara T. Usefulness of adiponectin to predict myocardial salvage following successful reperfusion in patients with acute myocardial infarction. Am J Cardiol 101: 1712-1715, 2008.

24. Shibata R, Sato K, Kumada M, Izumiya Y, Sonoda M, Kihara S, Ouchi N and Walsh K. Adiponectin accumulates in myocardial tissue that has been damaged by ischemia-reperfusion injury via leakage from the vascular compartment. Cardiovasc Res 74: 471-479, 2007.

\section{Shibata R, Sato K, Pimentel DR, Takemura Y, Kihara S, Ohashi K, Funahashi T, Ouchi N and} Walsh K. Adiponectin protects against myocardial ischemia-reperfusion injury through AMPK- and COX-2-dependent mechanisms. Nat Med 11: 1096-1103, 2005.

\section{Shimabukuro M, Higa N, Asahi T, Oshiro Y, Takasu N, Tagawa T, Ueda S, Shimomura I,} Funahashi T and Matsuzawa Y. Hypoadiponectinemia Is Closely Linked to Endothelial Dysfunction in Man. J Clin Endocrinol Metab 88: 3236-3240, 2003.

27. Shore SA, Terry RD, Flynt L, Xu A and Hug C. Adiponectin attenuates allergen-induced airway inflammation and hyperresponsiveness in mice. J Allergy Clin Immunol 118: 389-395, 2006. 
28. Tan KCB, Xu A, Chow WS, Lam MCW, Ai VHG, Tam SCF and Lam KSL. Hypoadiponectinemia Is Associated with Impaired Endothelium-Dependent Vasodilation. J Clin Endocrinol Metab 89: 765-769, 2004.

29. Tang YT, Hu T, Arterburn M, Boyle B, Bright JM, Emtage PC and Funk WD. PAQR proteins: a novel membrane receptor family defined by an ancient 7-transmembrane pass motif. J Mol Evol 61: 372-380, 2005.

30. Tao L, Gao E, Hu A, Coletti C, Wang Y, Christopher TA, Lopez BL, Koch W and Ma XL. Thioredoxin reduces post-ischemic myocardial apoptosis by reducing oxidative/nitrative stress. $\mathrm{Br} J$ Pharmacol 149: 311-318, 2006.

31. Tao L, Gao E, Jiao X, Yuan Y, Li S, Christopher TA, Lopez BL, Koch W, Chan L, Goldstein BJ and Ma XL. Adiponectin cardioprotection after myocardial ischemia/reperfusion involves the reduction of oxidative/nitrative stress. Circulation 115: 1408-1416, 2007.

32. Tao L, Gao E, Bryan NS, Qu Y, Liu HR, Hu A, Christopher TA, Lopez BL, Yodoi J, Koch WJ, Feelisch M and Ma XL. Cardioprotective effects of thioredoxin in myocardial ischemia and reperfusion: Role of S-nitrosation. PNAS 101: 11471-11476, 2004.

33. Wang QD, Pernow J, Sjoquist PO and Ryden L. Pharmacological possibilities for protection against myocardial reperfusion injury. Cardiovasc Res 55: 25-37, 2002. 
35. Wang Y, Tao L, Yuan Y, Lau WB, Li R, Lopez BL, Christopher TA, Tian R and Ma XL. Cardioprotective effect of adiponectin is partially mediated by its AMPK-independent antinitrative action. Am J Physiol Endocrinol Metab 297: E384-E391, 2009.

36. Xing Y, Musi N, Fujii N, Zou L, Luptak I, Hirshman MF, Goodyear LJ and Tian R. Glucose Metabolism and Energy Homeostasis in Mouse Hearts Overexpressing Dominant Negative \{alpha\}2 Subunit of AMP-activated Protein Kinase. J Biol Chem 278: 28372-28377, 2003.

37. Yamauchi T, Kamon J, Ito Y, Tsuchida A, Yokomizo T, Kita S, Sugiyama T, Miyagishi M, Hara K, Tsunoda M, Murakami K, Ohteki T, Uchida S, Takekawa S, Waki H, Tsuno NH, Shibata Y, Terauchi Y, Froguel P, Tobe K, Koyasu S, Taira K, Kitamura T, Shimizu T, Nagai R and Kadowaki T. Cloning of adiponectin receptors that mediate antidiabetic metabolic effects. Nature 423: 762-769, 2003.

\section{Zhu M, Miura J, Lu LX, Bernier M, DeCabo R, Lane MA, Roth GS and Ingram DK. Circulating} adiponectin levels increase in rats on caloric restriction: the potential for insulin sensitization. Exp Gerontol 39: 1049-1059, 2004. 


\section{Figure Legends}

463 Figure 1. Oral AdipoRon administration significantly improved $d P / d t_{\max }$ and $-d P / d t_{\max }$ in 464 wild type (WT), Adiponectin knockout $\left(\mathrm{APN}^{-/}\right)$and cardiomyocyte-specific AMPK- $\alpha_{2}$ 465 subunit mutant transgenic mice (AMPK-DN). $\mathrm{N}=14-16$ animals/group. ${ }^{*} \mathrm{P}<0.05, * * \mathrm{P}<0.01$ vs. $466 \mathrm{MI} / \mathrm{R}+$ vehicle. $\mathrm{P}$ values between $\mathrm{MI} / \mathrm{R}$ vs. sham are all less than 0.01 (not labeled, the same 467 for all figures).

Figure 2. Oral AdipoRon administration significantly enhanced left ventricular ejection 469 fraction $(\mathrm{EF} \%)$ determined by echocardiography in WT, $\mathrm{APN}^{-/-}$and AMPK-DN. Note attenuated response to AdipoRon treatment in AMPK-DN mice compared to WT mice. However, a significant portion of cardioprotection is retained in AMPK-DN mice. N=14-16 animals/group. ${ }^{*} \mathrm{P}<0.05,{ }^{*} * \mathrm{P}<0.01$ vs. $\mathrm{MI} / \mathrm{R}+$ vehicle.

Figure 3. Oral AdipoRon administration significantly attenuated post-ischemic cardiac apoptosis determined by DNA ladder formation (A) and caspase-3 activation (B) in WT and $\mathrm{APN}^{-/-}$mice. AdipoRon also significantly inhibited DNA ladder formation and reduced caspase-3 activity in AMPK-DN mice, albeit to lesser extent seen in WT mice. $\mathrm{N}=6-8$ animals/group. ${ }^{*} \mathrm{P}<0.05, * * \mathrm{P}<0.01$ vs. $\mathrm{MI} / \mathrm{R}+$ vehicle.

Figure 4. Oral AdipoRon administration significantly attenuated post-ischemic cardiac apoptosis determined by TUNEL staining in $\mathrm{WT}$ and $\mathrm{APN}^{-/-}$mice. AdipoRon also significantly inhibited TUNEL staining in AMPK-DN mice, albeit to lesser extent seen in WT mice. $\mathrm{N}=6-8$ animals/group. ${ }^{*} \mathrm{P}<0.05,{ }^{*} \mathrm{P}<0.01$ vs. $\mathrm{MI} / \mathrm{R}+$ vehicle.

Figure 5. AdipoRon activated AMPK signaling (determined by ACC phosphorylation) in WT, 
483 but not in AMPK-DN, animals (A). AdipoRon significantly inhibited ischemia/reperfusion 484 induced superoxide production, determined by DHE staining (B), lucigenin-enhanced 485 luminescence assay (C), and gp9 ${ }^{\text {phox }}$ overexpression (D) in AMPK-DN mice. $\mathrm{N}=6-8$ 486 animals/group for DHE staining and Western analysis; N=9-11 animals/group for 487 lucigenin-enhanced luminescence assay. ${ }^{*} \mathrm{P}<0.05, * * \mathrm{P}<0.01$ vs. $\mathrm{MI} / \mathrm{R}+$ vehicle. 

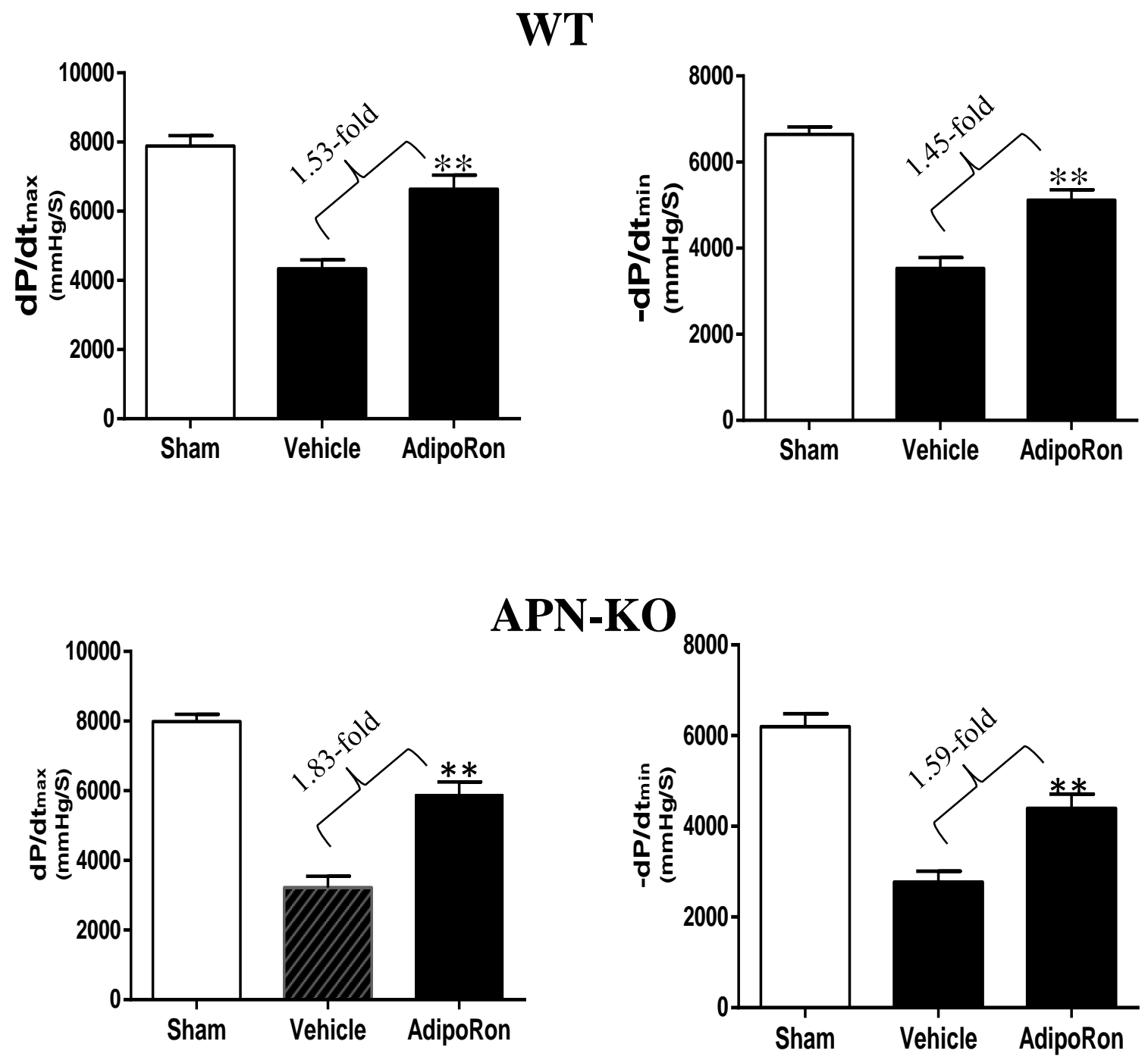

\section{AMPK-DN}
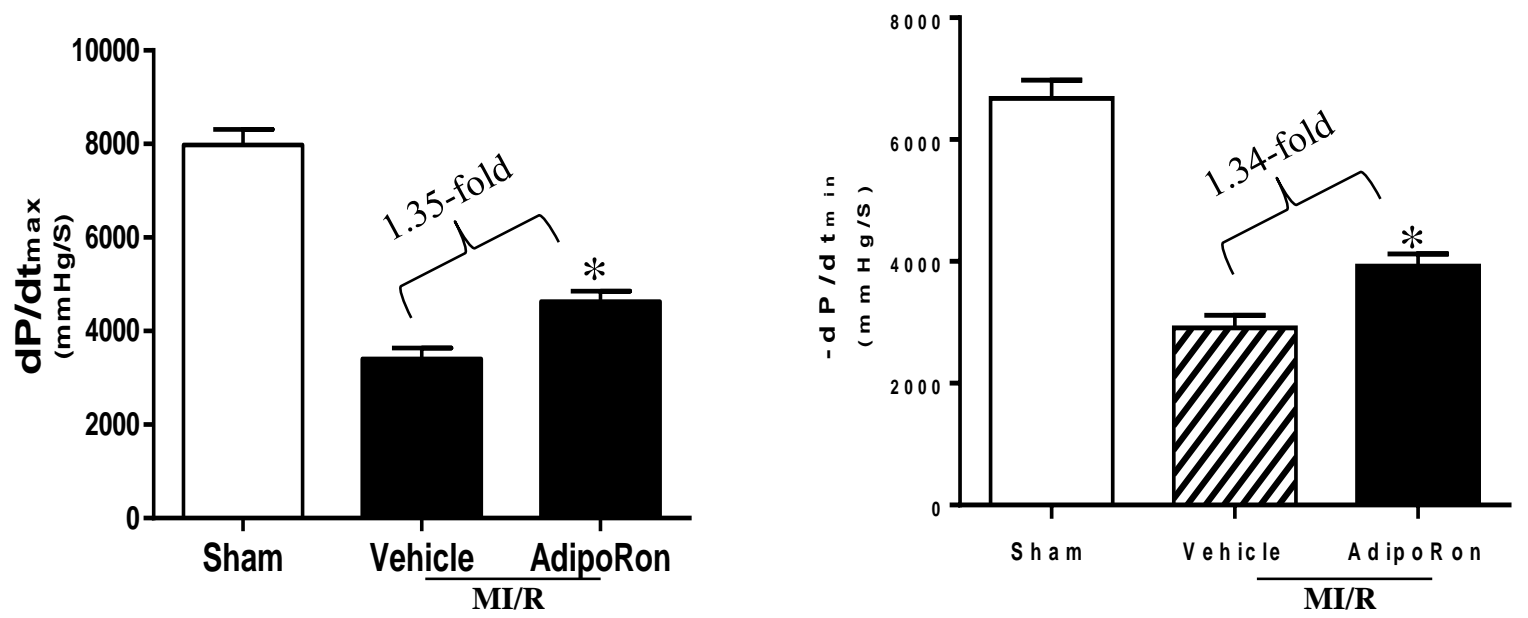

Figure 1 

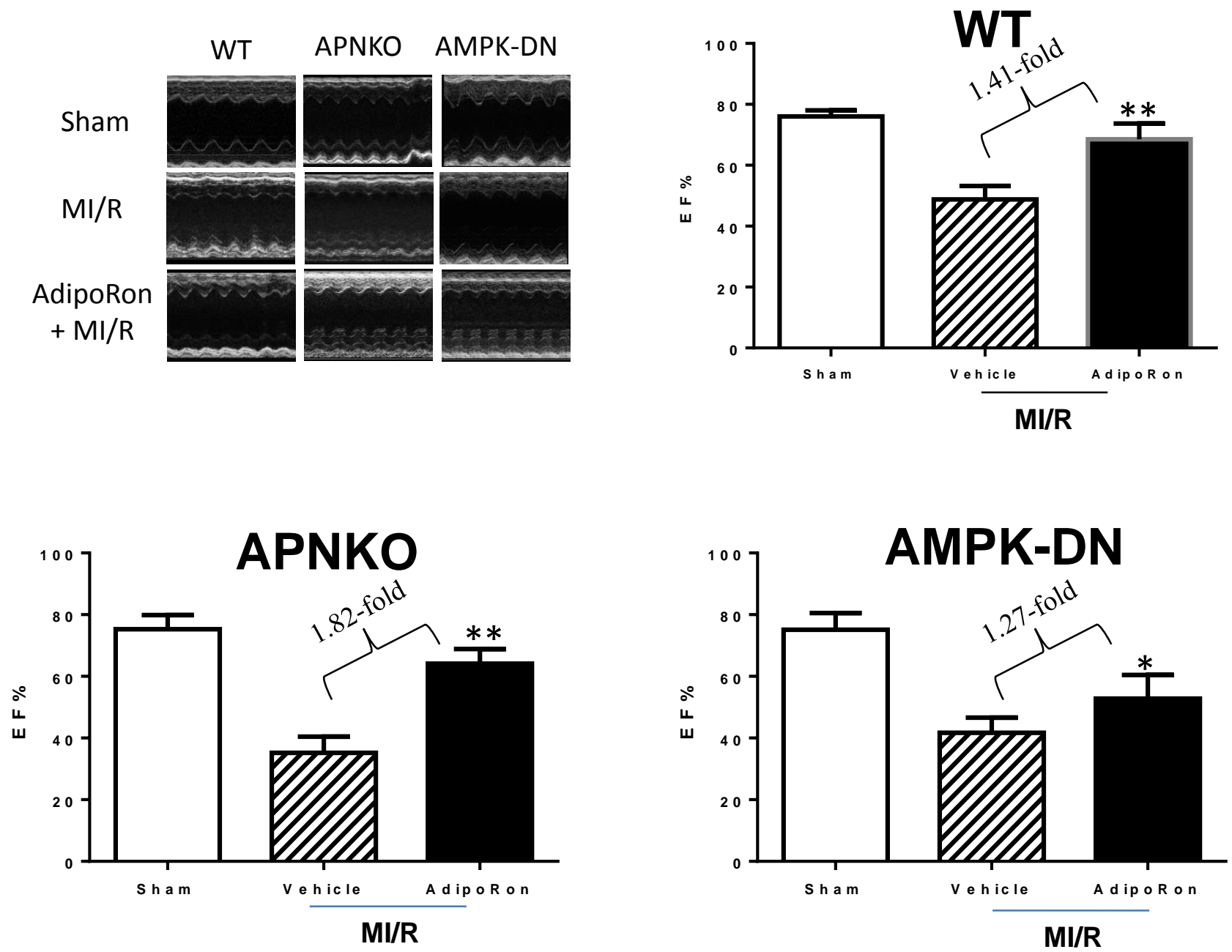

Figure 2 
A

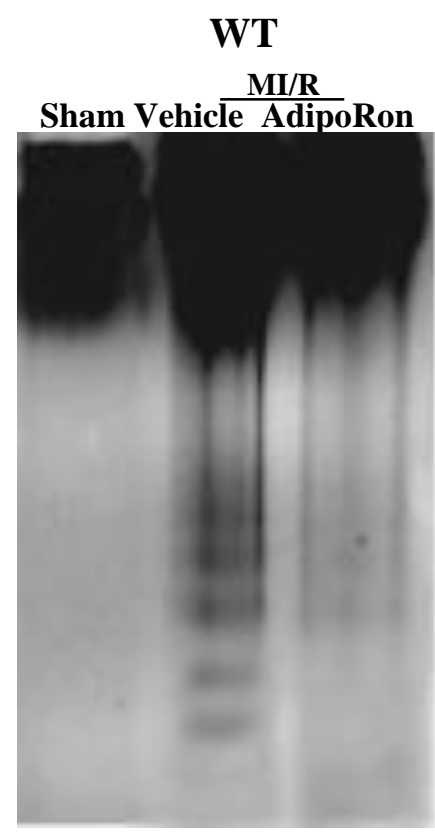

B

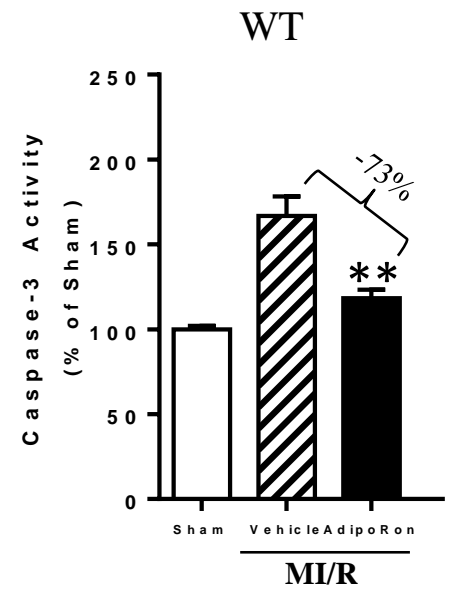

APNKO

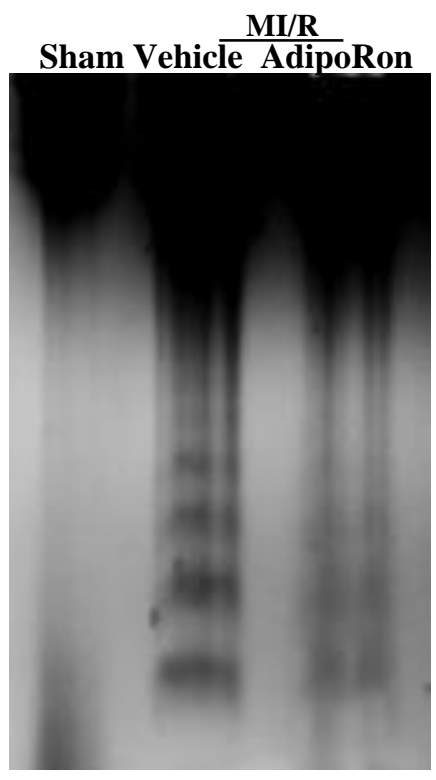

APNKO

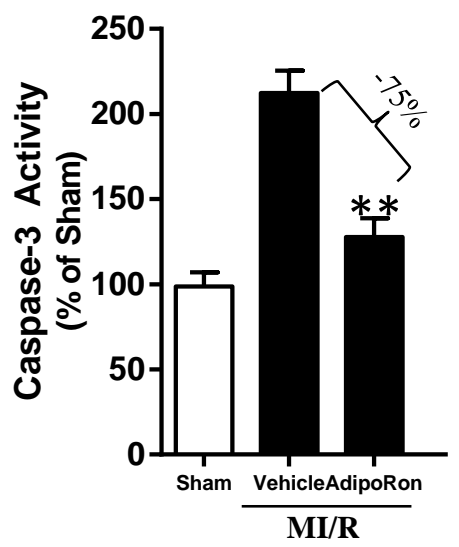

AMPK-DN

Sham Vehicle AdipoRon
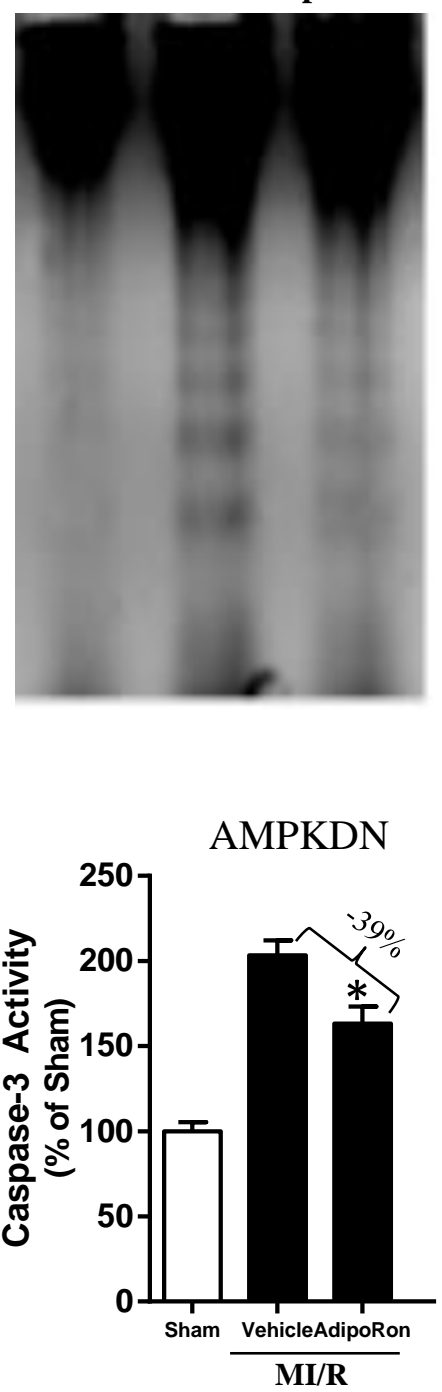

Figure 3 

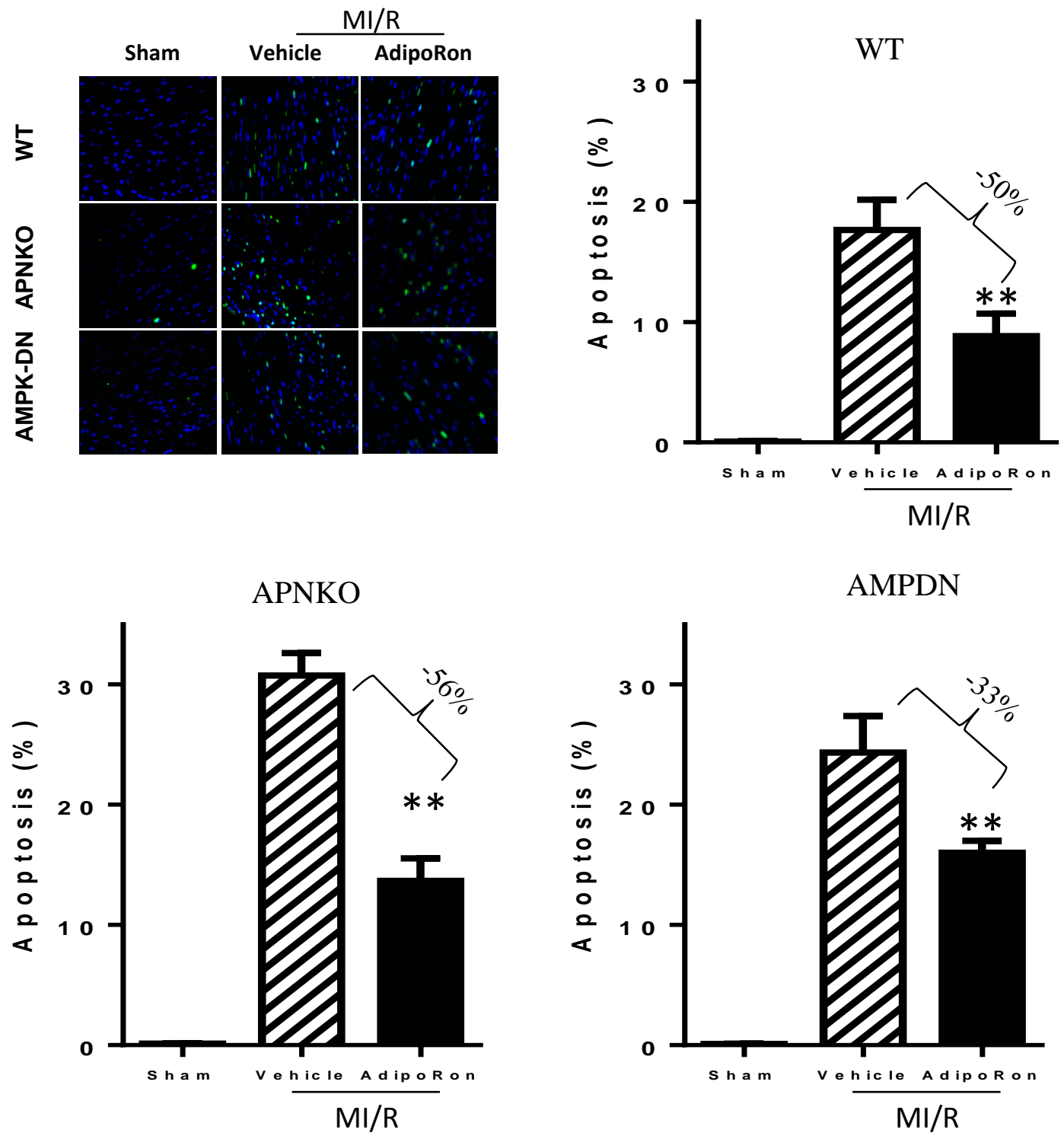

Figure 4 

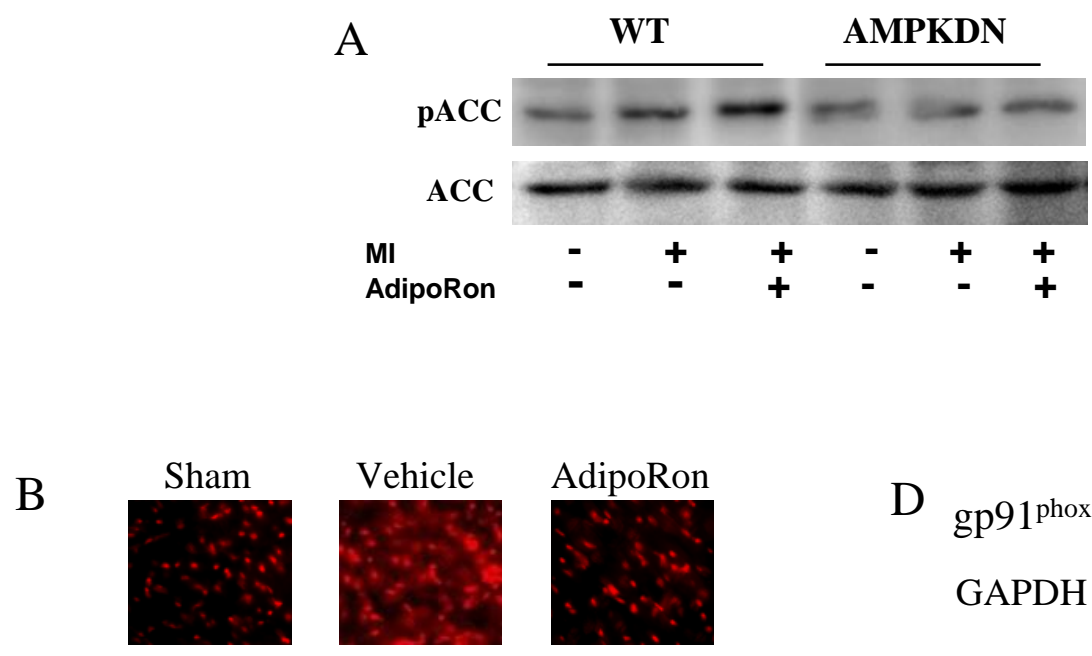

C
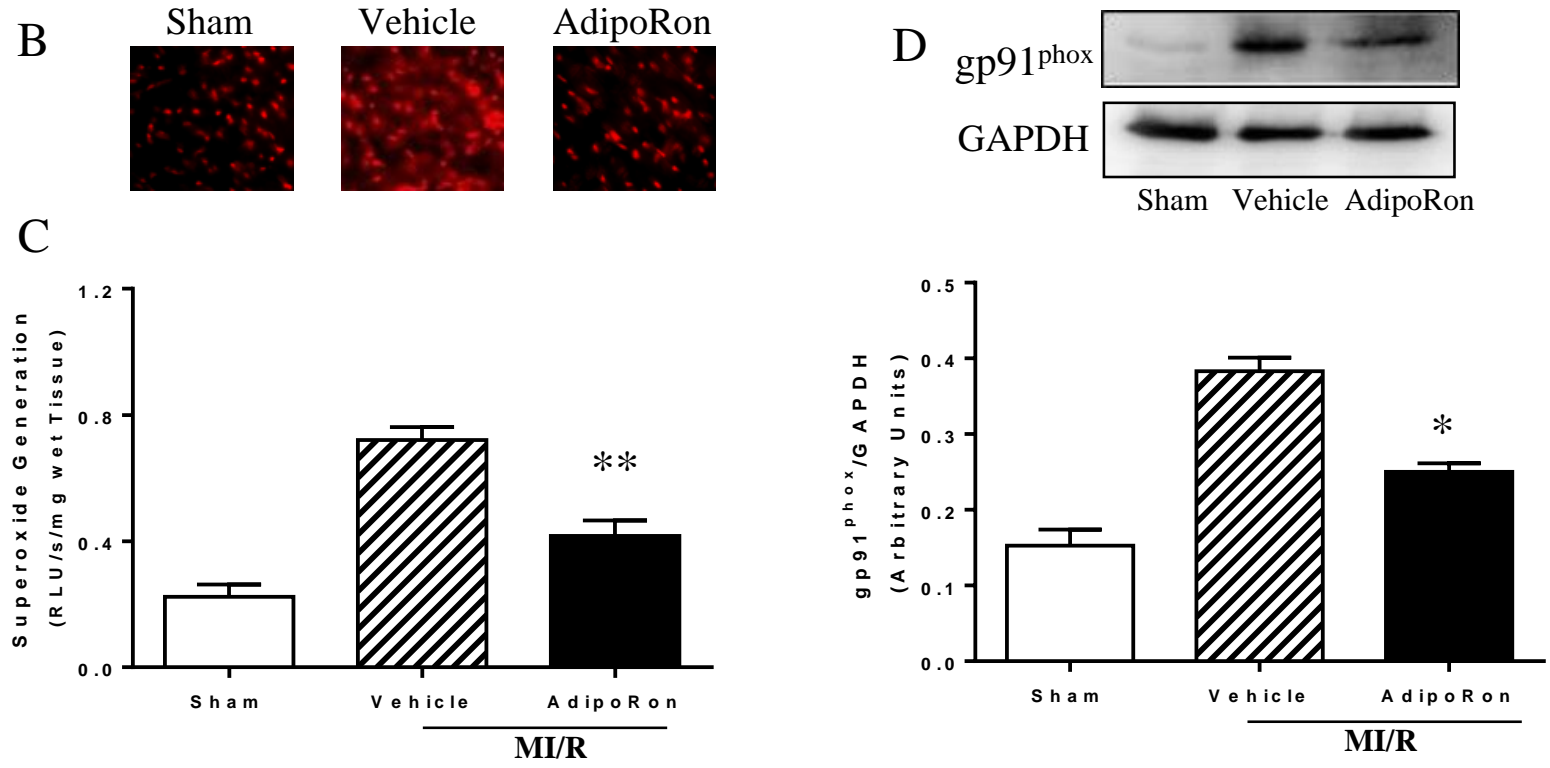

Figure 5 